\title{
Una propuesta pedagógica para el aprendizaje de la transformación climática de Chile en el contexto del cambio global
}

\author{
Osvaldo Sepúlveda Moreno ${ }^{1}$ \\ Recibido: 6 de julio de 2018 - Aprobado: 31 de agosto de 2018
}

\begin{abstract}
Resumen
En este artículo se presenta una propuesta pedagógica para los niveles de $5^{\circ}$ y $6^{\circ}$ básico, con el objetivo de rediseñar las actividades en la sala de clases sobre las implicancias del cambio climático en la configuración de los climas de Chile. Para ello se utiliza la nueva cartografía climatológica de Chile, siguiendo la metodología de Köppen-Geiger. Se postula, además, la necesidad de adoptar estas sugerencias no solo en la enseñanza de la asignatura de Historia, Geografía y Ciencias Sociales, sino también en aquellas actividades relacionadas con el Plan de Formación Ciudadana. Esta propuesta se fundamenta en la idea de que la aproximación en el aula al estudio del cambio climático debe ser considerada una oportunidad para que los docentes contextualicen el currículo escolar en la interacción con el entorno natural e incluyan otras formas de aprendizaje incorporando nuevas tecnologías y cartografías.
\end{abstract}

Palabras clave: cambio climático, climas de Chile, geografía, actividades en aula.

\section{A pedagogical proposal for learning about Chile's climate transformation in global change context}

\begin{abstract}
This article presents a pedagogical proposal for 5th and 6th grade levels aimed at redesigning classroom activities related to the implications of climate change in the configuration of Chilean climates. For this, the new Chilean climatological cartography is used following the Köppen-Geiger methodology. It also postulates the need to adopt these suggestions not only in the teaching of History, Geography and Social Sciences, but also in those activities related to the Citizenship Forming Plan. This proposal is based on the idea that the study of climate change in the classroom should be considered as an
\end{abstract}

1 Chileno. Profesor de Historia, Geografía y Educación Cívica, Universidad Metropolitana de Ciencias de la Educación, Chile; Magister en Geografía, Universidad de Chile. Centro Educacional Mariano Egaña, Peñalolén. E-mail: osvaldo.sepulvedamoreno@gmail.com 
opportunity for teachers to contextualize the school curriculum in interaction with the natural environment and include other forms of learning with new technologies and cartographies.

Keywords: climate change, Chilean climates, geography, classroom activities.

\title{
Uma proposta pedagógica para a aprendizagem da transformação climática do Chile no contexto da mudança global
}

\begin{abstract}
Resumo
Neste artigo apresenta-se uma proposta pedagógica para os níveis de $5^{\circ}$ e $6^{\circ}$ ano do ensino fundamental com o objetivo de redesenhar as atividades na sala de aula sobre as implicações da mudança climática na configuração dos climas do Chile. Para aquilo, utiliza-se a metodologia de Köppen-Geiger. Propõe-se, também, a necessidade de adotar estas sugestões não só no ensino da disciplina de História, Geografia e Ciências Sociais, mas também naquelas atividades relacionadas com o Plano de Formação Cidadã. Esta proposta fundamenta-se na ideia de que a aproximação ao estudo da mudança climática na sala de aula deve ser considerada uma oportunidade para que os professores contextualizem o currículo escolar na interação com o entorno natural e incluam outras formas de aprendizagem incorporando novas tecnologias e cartografias.
\end{abstract}

Palavras-chave: Mudança climática, climas do Chile, geografia, atividades na sala de aula.

La aplicación en las salas de clases en el ámbito del aprendizaje de las ciencias sociales siempre ha ido de la mano de la práctica y estudio de la historia. Con esta premisa, son muchas las personas que creen que la historia y la geografía son una sola; si bien esta es una concepción muy asimilada por los estudiantes de niveles básico y medio, es necesario generar su división bajo la idea de poder concretar espacios metodológicos muy disímiles entra una disciplina y otra. No podemos descartar la implicancia e importancia, pero sobre todo la integración, en nuestra vida diaria, de los sistemas de información cartográficos en diferentes elementos tecnológicos del devenir. Como sostiene De Miguel (2014), la penetración de la geoinformación ha ocurrido tanto en los hogares de los estudiantes como en sus teléfonos móviles, además de impactar "las herramientas, técnicas y procedimientos para la enseñanza de la geografía" (De Miguel, 2014: 21).

La literatura desarrollada sobre la relación entre geografía y ciudadanía se ha centrado en responder preguntas acerca de la utilidad del estudio de la geografía en la escuela y, específicamente, la contribución que la geografía pueda prestar a la formación ciudadana de los estudiantes, aunque desde diversas aproximaciones (Capel, 1998 y Souto, 2018). Capel afirma, por ejemplo, que el estudio de la geografía es valioso por tres razones: "1) porque ya existe y es 
una ciencia con una larga y rica tradición intelectual; 2) porque es una disciplina con un gran valor educativo y formativo, y 3) porque hay problemas que la geografía, es decir los profesionales formados en el campo de la geografía, puede ayudar a estudiar y resolver" (Capel, 1998). Souto, en tanto, se centra en "reflexionar sobre las decisiones administrativas para organizar el curriculum" y argumenta que la concepción del mundo que tienen profesores, quienes forman profesores en universidades y curriculistas, influye en estas decisiones.

En los últimos años la geografía ha experimentado diversos cambios en el currículum escolar de Chile. Como ha observado Gazmuri (2017), las tres últimas versiones del currículum chileno implementadas desde 1996 se han centrado en organizar de manera interdisciplinaria todos aquellos contenidos relativos a historia, sociedad y ciudadanía, con el objetivo de que "los estudiantes desarrollaran los conocimientos, habilidades y actitudes que les permitiesen una comprensión crítica de la sociedad y una valoración de la democracia y los derechos humanos" (Gazmuri, 2017: 158). Este enfoque interdisciplinario permite instalar una forma distinta de producir el conocimiento en los estudiantes, ya no se habla de verdad y entendimiento absoluto e indiscutible, puesto que hoy el discernimiento es relativo. Este hecho afectará a los estudiantes y docentes de geografía, ya que la evolución del aprendizaje entregado y la obtención de sus habilidades cognitivas les facultan para mirar el mundo de otra manera y reconstruir y comprender el espacio desde otras visiones posibles. La enseñanza de la geografía posibilita invitar a experimentar y construir una actitud de respeto a lo diferente y a sus representaciones espaciales, así como también entender y explicar algunos de los problemas de actualidad mundial compartiendo una mirada geográfica y más reflexiva.

La situación actual y su relación con el medio deben plantear reformadoras metodologías en la enseñanza de la ciencia geográfica e ir relacionándola con modernas propuestas de actividades de aulas que nacen en el área misma. La Sociedad Chilena de Ciencias Geográficas (SOCHIGEO) manifestó, en un informe del año 2013 sobre las Bases Curriculares de $7^{\circ}$ básico a $2^{\circ}$ medio, su inquietud porque el documento curricular comprende a la geografía como una ciencia auxiliar de la historia, desconociendo la evolución de ambas disciplinas en los últimos años. Se critica también que la geografía sea considerada solo por su trabajo cartográfico, desconociendo que éste es solo un medio de trabajo de la disciplina. Por último, se llama la atención a la necesidad de considerar a la geografía como una ciencia social. Para ello, la SOCHIGEO propone que el currículum nacional incorpore una progresión de las principales habilidades de la geografía, tales como:

"localización espacial, comparación entre lugares, asociación de patrones espaciales, interrelación y análisis del movimiento y difusión de elementos en el espacio geográfico, interpretación de datos sociales, interpretación de cartografía regular y temática, imágenes aéreas y de 
satélites, utilización de gráficos y uso de nuevas tecnologías para el análisis territorial y, especialmente, la toma de decisiones" (Sociedad Chilena de Ciencias Geográficas, 2013).

Todos estos antecedentes permiten afirmar que innovar en prácticas metodológicas y nuevas actividades en el aula desde la enseñanza de la geografía se hace vital para nuestros tiempos. Es por esto que concordamos con De la Calle (2012) en que la geografía enseñada en los colegios no puede centrarse solo en la localización, sino que el estudiante debe conocerla también como una ciencia explicativa. En el presente artículo, no solo abordaremos la situación del curriculum nacional para enseñanza básica en el caso de la asignatura de Historia, Geografía y Ciencias Sociales, sino que sumaremos además el Plan de Formación de Ciudadana. De esta forma los estudiantes podrán incorporar a sus aprendizajes no solo aspectos pragmáticos, tales como localizar un lugar adecuado de vacaciones, guiarse con un mapa en una ciudad o entender un plano donde aparecen los usos del suelo urbano. También queremos hacer hincapié en la capacidad de esta materia para plantear cuestiones relativas a la manera de organizar el espacio de dos diferentes agentes sociales: entorno natural y cambio climático en un contexto en que se relacionan ciudad, problemática urbana y educación (Soto, 2010).

Por otra parte, en la actualidad el cambio climático es un tema país. En las escuelas nacionales debe ser tratado y sociabilizado dentro de los Proyectos Educativos Institucionales (PEI), Plan de Mejoramiento Educativo (PME), Plan Integral de Seguridad Escolar (PISE), Plan de Formación Ciudadana y otros instrumentos de gestión establecidos por el Ministerio de Educación de la manera que cada establecimiento estime, como a nivel de misión o visión, o bien como un sello educativo de mejora continua. Los cambios de temperatura durante el siglo XX muestran considerables variaciones temporales (taza de cambio variable en el tiempo y superpuesta en fluctuaciones interanuales muy importantes) y espaciales (Garreaud, 2011). En el nivel escolar, desde primer ciclo a enseñanza media, los docentes de geografía deben centrar la explicación de esta transformación en un cambio constante del clima cada treinta años, y definido este mismo como un promedio de largo plazo de los elementos de temperatura, humedad, precipitación, presión atmosférica y vientos. De esta forma, en cada colegio de Chile, referente a su entorno natural, se podrían establecer condiciones climáticas diferentes, serán encontradas circunstancias y variables naturales específicas que afectan la vida de los estudiantes, así como actividades que emprenden las personas en el mismo espacio. Los estudiantes podrían entender los efectos del cambio climático en la vida de cada uno de ellos en el presente y futuro, circunscribirse a su propio espacio natural, para lograr una mejor enseñanza, aprendizajes y comprensión del contenido, buscando este ser un elemento que unifique criterios, despierte una evaluación centrada principalmente en 
el pensamiento crítico y análisis de la situación actual del cambio climático. Por tanto, se hace relevante comprender estas transformaciones climáticas desde el entorno natural de cada uno de los estudiantes para poder acercar el conocimiento a la vida diaria.

Dado que, como han demostrado Sarricolea et al. (2017), es importante comprender cómo la relación entre el efecto del cambio climático y la distribución de los tipos de clima tiene directa incidencia en la planificación de las actividades económicas de una determinada región, es necesario que los docentes puedan comprender y transmitir a sus estudiantes, en el entorno que se encuentren ejerciendo la docencia, que se están produciendo, y seguirán, mutaciones en sus regiones agrícolas, forestales, costeras, desérticas, vitivinícolas, entre otras. También, los docentes deberán deducir, con el uso de la cartografía y la tecnología recientes, clasificaciones climáticas basadas en escenarios futuros y las transformaciones climáticas observadas y proyectadas en la vida cotidiana, las cuales estarán representadas por el mapa de la clasificación climática de Köppen-Geiger. Sarricolea et al. (2017) también destacan la existencia de investigaciones que señalan lo trascendental y valioso de este uso de mapas tanto para la investigación científica como para la educación.

La clasificación climática de Köppen-Geiger es una estructura de orden jerárquico. Esta clasificación climática es algebraica, pues considera valores numéricos y proporciones, y también es empírica, ya que estima umbrales que condicionan la distribución de la vegetación, por tanto, hace uso de un sistema de letras mayúsculas y minúsculas que denotan rasgos particulares de los climas. Esto se muestra en las tablas 1 y 2 . En relación con los cambios climáticos de toda la superficie nacional en los últimos 45 años, investigadores han realizado una nueva distribución climática (Sarricolea et al., 2017). Esto significa que se afecta también el proceso de enseñanza en las aulas del país.

Tabla 1

Elementos considerables por Köppen-Geiger para su clasificación climática

\begin{tabular}{|l|l|l|}
\hline Temperatura & Precipitación & Vegetación \\
\hline A tropical & f: todo el año & s: estepa \\
\hline B seco & $\begin{array}{l}\text { m: tipo monzón } \\
\text { (abundante en verano) }\end{array}$ & w: desierto \\
\hline C templado & w: en verano & t: tundra \\
\hline D frío & s: invierno & b: alta montaña \\
\hline E polar & & f: hielos perpetuos \\
\hline
\end{tabular}

Fuente: Köppen-Geiger, 1900a y 1900b. 
Tabla 2

Clasificación climática de Köppen-Geiger

\begin{tabular}{|c|c|c|c|c|}
\hline $\begin{array}{l}\text { Climas } \\
\text { tropicales }\end{array}$ & Climas secos & $\begin{array}{l}\text { Climas } \\
\text { templados }\end{array}$ & Climas fríos & Climas polares \\
\hline $\begin{array}{l}\text { Af : tropical } \\
\text { con lluvias } \\
\text { todo el año }\end{array}$ & $\begin{array}{l}\text { Bs : seco } \\
\text { estepárico }\end{array}$ & $\begin{array}{l}\text { cf: templado } \\
\text { con Iluvias } \\
\text { todo el año }\end{array}$ & $\begin{array}{l}\text { Df: fríos con } \\
\text { lluvias todo el } \\
\text { año }\end{array}$ & $\begin{array}{l}\text { Et : polar de } \\
\text { tundra }\end{array}$ \\
\hline $\begin{array}{l}\text { Aw: tropical } \\
\text { con lluvias en } \\
\text { el verano }\end{array}$ & \multirow[t]{2}{*}{$\begin{array}{l}\text { Bw seco } \\
\text { desértico }\end{array}$} & $\begin{array}{l}\text { Cw : templado } \\
\text { con lluvias en } \\
\text { verano }\end{array}$ & \multirow[t]{2}{*}{$\begin{array}{l}\text { Dw: frío con } \\
\text { lluvias en } \\
\text { verano }\end{array}$} & $\begin{array}{l}\text { Ef: polar } \\
\text { de hielos } \\
\text { perpetuos }\end{array}$ \\
\hline $\begin{array}{l}\text { Am: tropical } \\
\text { con lluvias } \\
\text { monzónicas }\end{array}$ & & $\begin{array}{l}\text { Cs: templado } \\
\text { con lluvias en } \\
\text { invierno }\end{array}$ & & $\begin{array}{l}\text { Eb : polar de } \\
\text { alta montaña }\end{array}$ \\
\hline
\end{tabular}

Fuente: Köppen-Geiger, 1900a y 1900b.

Por otra parte, el Ministerio del Medio Ambiente publicó el año 2012 una guía que facilita el progreso de los "conceptos, actitudes y habilidades necesarias para ejercer una ciudadanía informada y responsable frente al fenómeno del cambio climático" (Ministerio del Medio Ambiente, 2012: 47). Mediante formas de aprendizaje que incluyan nuevas tecnologías y cartografías, y acerquen estudios e investigaciones universitarias a los profesores, se afirma en el documento gubernamental, se podría insertar la comprensión y dedicación que merece el tema del cambio climático en el aula. De esta manera, los docentes serían capaces de desplegar de una mejor manera el curriculum nacional, "acercándolo a los estudiantes del siglo XXI con temáticas motivadoras, estimulantes y fundamentales para su futuro personal, como de su localidad, región, país y de todos quienes vivimos en este planeta" (Ministerio del Medio Ambiente, 2012: 48).

\section{Materiales y Métodos}

La investigación planteada tiene como área de estudio el territorio chileno, escogido sobre la base de la nueva cartografía climatológica de Chile, siguiendo la metodología de Köppen-Geiger realizada por Sarricolea, Herrera, y Meseguer (2016). Se utilizará el actual curriculum nacional y los programas de estudio vigentes en Chile correspondientes a los niveles $5^{\circ} \mathrm{y}$ $6^{\circ}$ básico de la asignatura de Historia, Geografía y Ciencias Sociales, con el objetivo de crear propuestas para integrar la nueva cartografía climática de Chile. Incluyendo, además, las disposiciones del nuevo Plan de Formación Ciudadana propuestas para el año 2017 y la guía de apoyo docente sobre cambio climático del Ministerio del Medio Ambiente, el objetivo de nuestra 
propuesta es facilitar a los docentes de todos los sectores del aprendizaje, la incorporación del tema del cambio climático en el currículo formal del segundo ciclo de la educación básica.

El trabajo consta de una sugerencia metodológica para los docentes que logren insertar en la enseñanza de la geografía esta nueva clasificación climática de Chile. El colegio en el cual se utilizará la implementación de las sugerencias a las mejoras de las actividades y del tema en el plan de Formación Ciudadana será el Centro Educacional Mariano Egaña, perteneciente a la Corporación Municipal de Peñalolén.

La cartografía del territorio utilizada corresponde al Mapa de Clasificación de Köppen-Geiger de Chile de Sarricolea et al. (2017), dependiente del Laboratorio de Climatología del Departamento de Geografía de la Universidad de Chile. ${ }^{2}$

2 La imagen del mapa original se puede revisar en:

https://www.tandfonline.com/doi/full/10.1080/17445647.2016.1259592?scroll=top\&nee dAccess=true, revisado el 5-V-2018. 
Figura 1

Zonas Climáticas en Chile

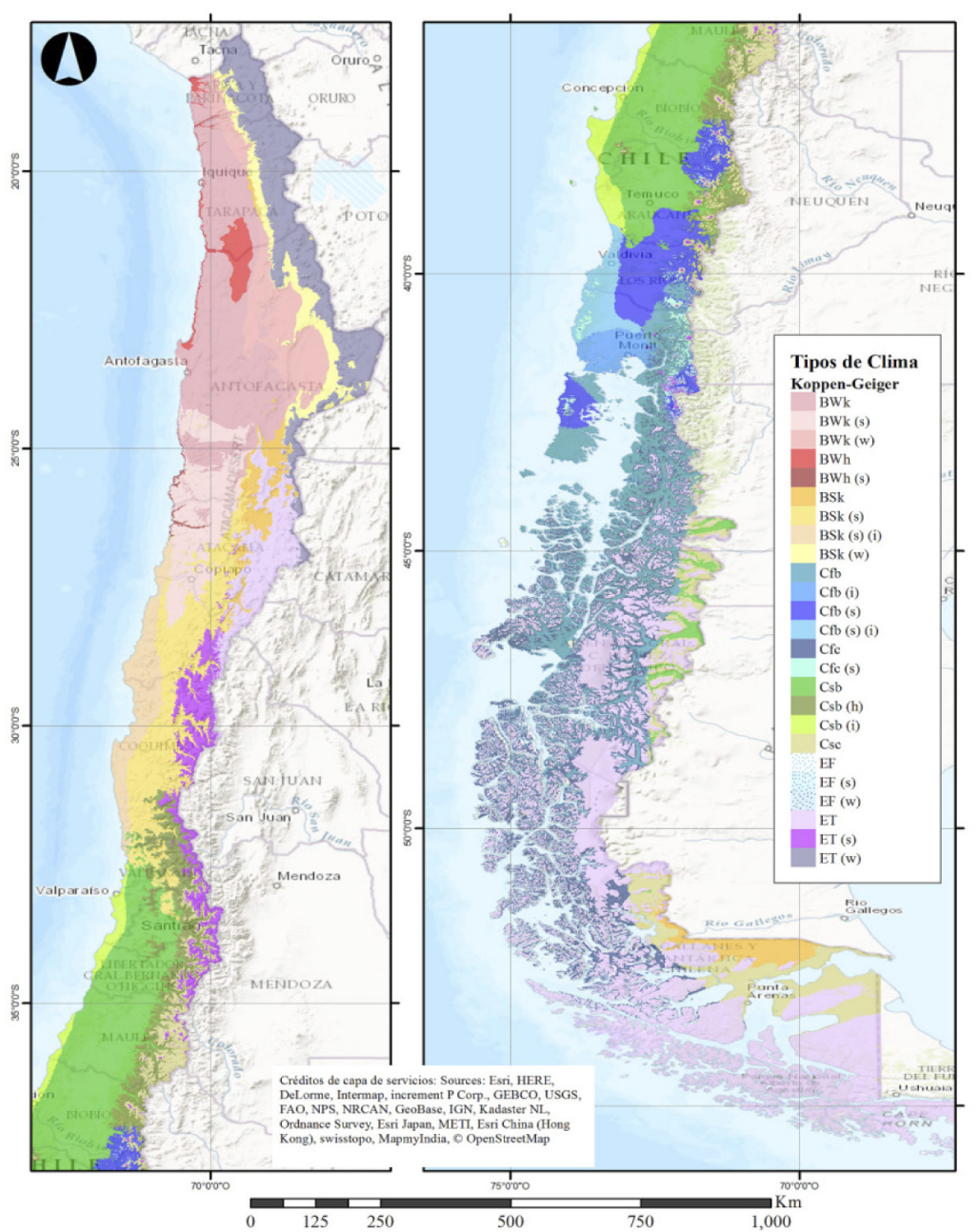

Fuente: Sarricolea et al. (2017).

\section{Resultados}

\section{Aspectos propios de la disciplina para conocer en el aula}

La investigación de Sarricolea et al. (2017), que establece la nueva clasificación climática de Chile, también señala que 
"el uso de superficies climáticas permite la generación de mapas con clasificaciones discretas e índices climáticos que, contrastados con datos de estaciones meteorológicas, facilitan una mejor aproximación de los límites y de la distribución espacial de los climas que en estudios previos. [Además], las singularidades detectadas permiten identificar 25 tipos de clima para el Chile continental basado en los criterios climáticos y geográficos habituales. Los criterios utilizados fueron: concentración y origen de las precipitaciones, las condiciones de isoterma, el comportamiento oceánico atenuado de las temperaturas y la elevación de $1000 \mathrm{~m}$ sobre el nivel del mar" (Sarricolea et al., 2017: 72-73). ${ }^{3}$

Estos principios entregan una mirada del territorio nacional más completa y detallada de transformaciones en algunas zonas, de acuerdo con el proceso de cambio climático, y que pueden ser estudiadas preferentemente en criterio de precipitaciones y temperaturas para cada región de Chile.

\section{Aplicación de metodologías efectivas para el aula y docentes}

Es necesario plantear que toda transformación y el uso de nuevas herramientas tecnológicas se transforma en un aprendizaje significativo para los y las estudiantes de todo docente comprometido con la educación actual. Asimismo, es preciso destacar que "las personas al adquirir esa información científica cambiarán su comportamiento, actitudes y valores, y se convertirán en una ciudadanía ambientalmente alfabetizada" (González y Meira, 2009: 10).

Los programas educativos del Ministerio de Educación cuentan con las unidades y actividades que representan la didáctica de los objetivos de aprendizaje, habilidades y actitudes plasmados en el currículum nacional. En el caso específico del cambio climático, además, está la necesidad de darle al tema una mirada integradora. Por ello, el desarrollo de competencias relativas tanto a la comprensión como a la acción sobre el cambio climático "es un desafío abierto a todos los sectores del aprendizaje" (Ministerio del Medio Ambiente, 2012: 47). La secretaría de Estado también advierte de la necesidad de abordar el cambio climático mediante una forma que "integre los saberes, habilidades y actitudes que aportan los diversos campos disciplinarios que constituyen el currículo escolar". También llama la atención que aproximarse en el aula al estudio del cambio climático es una oportunidad para los docentes "de contextualizar el currículo escolar en la interacción

3 Traducción propia. 
con la realidad, preocupaciones y sueños de niños, niñas y jóvenes". En un ámbito más general, concluye el Ministerio, no hay que olvidar que:

"la experiencia escolar debe ser un espacio que además del desarrollo de aprendizajes en los estudiantes, permita la reflexión comunitaria acerca del tipo de incremento al que se aspira, incorporando temáticas pertinentes a los problemas y desafíos actuales y fuertemente significativas en el desarrollo personal y social de quienes participan del proceso formativo" (Ministerio del Medio Ambiente, 2012: 48).

Es en este contexto que se debe entender que diversos planes de formación ciudadana incluyan, como una formación transversal, las transformaciones del cambio climático: en este caso específico presentaremos dos opciones de uso de una actividad y un plan de formación ciudadana adecuados a la temática de la transformación geográfica de Chile en un contexto de cambio climático.

A continuación se presentan tres propuestas metodológicas didácticas, dos para ser aplicadas en las aulas del sistema escolar y una para aplicar en el plan de formación ciudadana. En primer lugar se presentarán las actividades sugeridas en los programa de estudio de la asignatura de Historia, Geografía y Ciencias Sociales para $5^{\circ}$ y $6^{\circ}$ básico del Ministerio de Educación, dando cuenta de la carencia de temáticas relacionadas con el cambio climático, tensiones y análisis del espacio geográfico. ${ }^{4}$ En una segunda parte se mostrarán diseños y propuestas didácticas que tienen como foco principal el estudio del cambio climático y la utilización del mapa de la nueva clasificación climática siguiendo la metodología de Köppen-Geiger, para potenciar los procesos de enseñanza y aprendizaje de la geografía.

\section{Actividad 1}

Nivel: $5^{\circ}$ básico ${ }^{5}$

Objetivo de Aprendizaje 9: Caracterizar las grandes zonas de Chile y sus paisajes (Norte Grande, Norte Chico, Zona Central, Zona Sur y Zona Austral), considerando ubicación, clima (temperatura y precipitaciones), relieve, hidrografía, población y recursos naturales, entre otros.

Habilidad: Pensamiento espacial y trabajo con fuentes.

4 El programa de Historia, Geografía y Ciencias Sociales propone otras actividades para la unidad, pero las que hemos seleccionado guardan relación con el aporte de la implementación de un nuevo mapa de las zonas climáticas de Chile.

5 Ministerio de Educación, 2013a: 63-65. 


\section{Algunas actividades propuestas por Programa de Estudio Historia, Geografía y Ciencias Sociales Quinto año básico Unidad 1}

1. Los estudiantes visitan y observan paisajes que los rodean. Luego observan y analizan fotografías de diferentes paisajes de Chile y, apoyándose en sus conocimientos previos, responden preguntas tales como: ¿qué paisajes puedes distinguir en las fotografías? ¿Por qué crees que en Chile existen tantos paisajes diferentes? ¿Qué formas de relieve puedes observar en las fotografías? ¿Alguna de las fotografías se asemeja a la localidad dónde vives? Escriben sus respuestas en su cuaderno y las comparten con sus compañeros.

2. Construyen un cuadro resumen con las principales características de relieve, hidrografía, clima y vegetación de cada zona natural.

3. Describen, de modo general, el comportamiento que tienen las precipitaciones y las temperaturas a lo largo del territorio nacional, así como las características generales de las macroformas del relieve.

Algunas observaciones al docente: es relevante que para el conjunto de actividades que cubre este Objetivo de Aprendizaje se resalten de forma conjunta los aspectos que conforman los paisajes de Chile. Al igual que en años anteriores, es de suma importancia que la caracterización de estos elementos no se convierta en la memorización de las características físicas y humanas de las regiones, sino en la interconexión de estas y en cómo conforman espacios geográficos complejos y particulares.

- Los sitios http://siit2.bcn.cl/nuestropais/index_html, de la Biblioteca del Congreso Nacional y http://www.igm.cl/, del Instituto Geográfico Militar (IGM) brindan una amplia gama de recursos educativos útiles para el desarrollo de los Objetivos de Aprendizaje relativos a Geografía, con material teórico y visual.

- El sitio http://www.profesorenlinea.cl/Chilegeografia/GeografiaChile.html presenta una introducción breve sobre los elementos más importantes que componen la geografía de Chile.

- Para obtener imágenes, videos e información relativa a distintas zonas naturales de Chile, visitar http://www.chile.travel/es.

Respecto de la vinculación de temáticas contingentes de la enseñanza de la geografía y el currículum nacional, en el Objetivo de Aprendizaje no aparece ninguna proximidad al tema de cambio climático o zonas climáticas de Chile actualizadas. De acuerdo a esto, debemos entender que el docente tiene libertad para buscar su material educativo, pero si decide optar por las recomendaciones del mismo programa, no encontramos actualizaciones del tema a tratar en la sala de clases. Por ello nuestra proposición es que el docente busque material de trabajo que informe de las nuevas transformaciones de las zonas climáticas del territorio chileno y lo aplique en propuestas didácticas en la sala de clases. 


\section{Actividad propuesta}

La siguiente actividad se puede llevar a la práctica en un contexto de aula de la asignatura de Historia, Geografía y Ciencias Sociales, desde su secuencia didáctica, y requiere de 2 a 4 horas pedagógicas.

Inicio: El docente presenta en la sala de clases un mapa de la nueva clasificación climática siguiendo la metodología de Köppen-Geiger, informando de los "aspectos dinámicos" que posee el espacio geográfico y sus representaciones cartográficas.

Luego de la presentación, los estudiantes deben ubicar en el mapa las principales zonas naturales del país, identificando los rasgos físicos más distintivos de cada una de las zonas.

A su vez, explican por escrito por qué consideraron esos rasgos como los más distintivos, la diversidad de climas en las zonas naturales y mencionan otras características que también son relevantes.

Desarrollo: En la sala de clases se presentarán diferentes paisajes en cada una de las zonas naturales (como muestra el ejemplo de la figura 2). Las fuentes deben ser analizadas por los estudiantes, respondiendo las siguientes preguntas:

a) ¿Por qué existen diferentes climas en las zonas naturales?

b) ¿Cuál es la influencia del cambio climático en las transformaciones del espacio geográfico?

Figura 2

Antes y después del glaciar de Echaurren, Región Metropolitana de Santiago

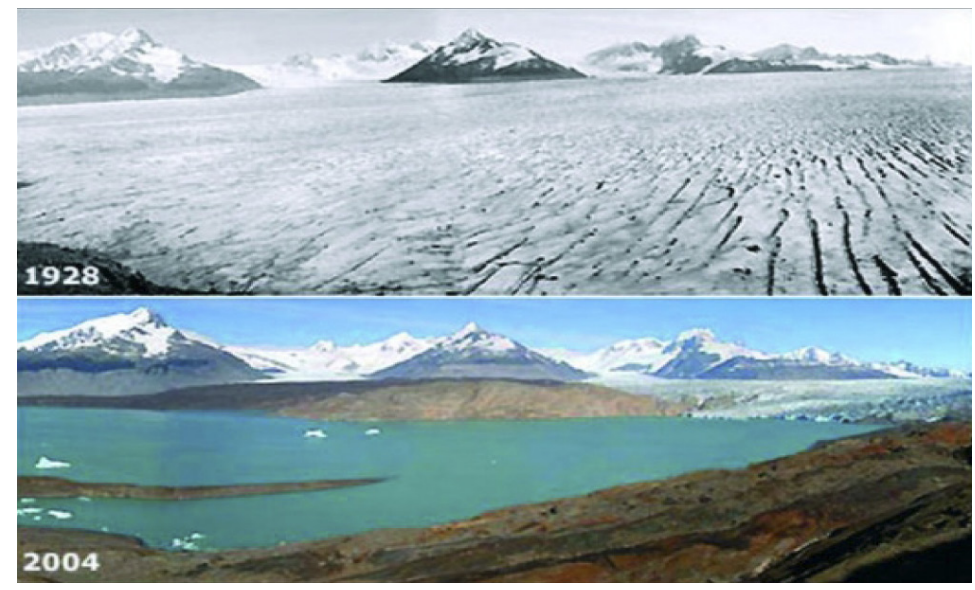

Fuente: www.plataformaurbana.cl, revisado el 30-VI-2018 
Cierre: Finalmente, los estudiantes deben redactar un breve ensayo (evaluación interdisciplinaria que relaciona las asignaturas de Lenguaje, Artes Visuales y Educación Tecnológica) en el que señalan las implicaciones multiespaciales y factoriales que afectan su vida diaria con el cambio climático presentado en la actualidad. El ensayo debe seguir el orden de un análisis escalar del espacio geográfico

Ejemplo:

Local: Barrio > Regional: Región > Nacional: Chile > Continental: América $>$ Global: Mundo

Recomendaciones: Este modelo de actividad en relación al mapa climático de Chile siguiendo la metodología de Köppen-Geiger, debe ser usado en trabajo de articulación docente (Ander-Egg, 1997) para lograr flexibilidad curricular en el tratamiento del contenido referente a cambio climático. De esta manera, a través de los climas, se instalará una nueva visión social, cultural y geográfica sobre las transformaciones climáticas actuales del territorio nacional.

\section{Actividad 2}

\section{Nivel: $6^{\circ}$ básico ${ }^{6}$}

Objetivo de Aprendizaje: Caracterizar geográficamente las regiones políticoadministrativas del país, destacando los rasgos físicos (como clima, relieve, hidrografía y vegetación) y humanos (como volumen y distribución de la población y actividades económicas) que les dan unidad.

Habilidad: Pensamiento espacial, trabajo con fuentes, pensamiento crítico, comunicación.

\section{Algunas actividades propuestas por Programa de Estudio Historia, Geografía y Ciencias Sociales Sexto año básico Unidad 4}

1. Con base en fuentes dadas por el docente y organizados en grupos, los estudiantes obtienen información sobre las características físicas de una región de Chile, considerando: > hidrografía (ríos, lagos, lagunas, glaciares) > clima (precipitaciones y temperaturas promedio) > vegetación predominante > nombres de formas del relieve características. Representan y organizan la información en mapas, esquemas, tablas y gráficos, y luego comparan los resultados de las distintas regiones estudiadas. Escriben una reflexión en su cuaderno acerca de cómo los factores físicos inciden en la unidad y delimitación de cada región.

6 Ministerio de Educación, 2013b: 171, 172, 173. 
2. Seleccionan una región de Chile y realizan una pequeña entrevista en la que preguntan a adultos cercanos y a su familia: ¿qué caracteriza mejor la región seleccionada? ¿Qué paisajes y recursos son característicos de esa región? Luego, a través de fuentes pertinentes, investigan acerca de: > sus paisajes característicos , el recurso natural más importante de esa región > las actividades económicas asociadas a este recurso > los trabajos asociadas a las actividades y las personas que los desempeñan.

Algunas observaciones al docente: La página de la Dirección meteorológica de Chile y de la Dirección General de la Aeronáutica Civil presentan información relevante respecto de los climas en Chile, en especial de cada región: http://www.meteochile. gob.cl/climas/climas.html

El sitio desarrollado por el Departamento de Geofísica (DGF) de la Facultad de Ciencias Físicas y Matemáticas (FCFM) de la Universidad de Chile aporta información sobre climas, meteorología y fenómenos asociados. http://www.atmosfera.cl/html/temas/ temas_02.html

El programa de Historia, Geografía y Ciencias Sociales propone otras actividades para la unidad, pero se han seleccionado las anteriores porque guardan relación con el aporte de la implementación de un nuevo mapa de las zonas climáticas de Chile. En el Objetivo de Aprendizaje no aparece ninguna proximidad al tema de cambio climático o zonas climáticas de Chile actualizadas. Sin embargo, es muy importante destacar las recomendaciones al docente, ya que en este caso se sugiere recurrir al Departamento de Geofísica de la Universidad de Chile, centro de estudios donde se concentra gran información de investigación sobre el tema de cambio climático y climatología de Chile. En otras palabras, se aproxima al docente a material actualizado con el cual pueda trabajar en la sala de clases. En este espacio se recomienda también la página del Laboratorio de Climatología del Departamento de Geografía de la Universidad de Chile, donde se encuentran recursos educativos, como el mapa de la nueva de la clasificación climática de Chile siguiendo la metodología de Köppen-Geiger, y otras informaciones de interés al docente. De esta forma, repetimos la reflexión anterior referente a que, si bien el docente tiene libertad para buscar su material educativo, si decide optar por las recomendaciones del mismo programa no encontramos actualizaciones del tema a tratar en la sala de clases detalladamente y siguiendo metodologías pedagógicas. Es importante conocer los datos, pero sin duda es más importante saber qué hacer con los datos, y cómo estos son entregados a los estudiantes para su justo conocimiento, comprensión y análisis. 


\section{Actividades propuestas}

\section{Primera actividad}

Inicio: los estudiantes reciben por parte del docente el mapa con la nueva clasificación climática de Chile continental siguiendo el modelo de KöppenGeiger, confeccionado por Sarricolea, Herrera y Meseguer (2017), y el mapa de Climas de Chile, confeccionado por el Instituto Geográfico Militar. Estos dos mapas deben ser presentados en Power-Point para el desarrollo visual de los mismos. Además, se sugiere incluir mapas para que así la actividad tenga una explicación visual.

Desarrollo: Los estudiantes deben confeccionar una tabla comparativa de análisis, sobre la base de cambios que se observen en las clasificaciones climáticas según macrozona geográfica: Chile norte, central, sur y austral. En esta comparación, el profesor puede incluir diferentes criterios, tales como clima, temperaturas, precipitaciones, vegetación, entre otros aspectos.

\section{Tabla 3}

Ejemplo de cuadro de análisis comparativo

\begin{tabular}{|l|l|l|}
\hline Criterio & Modelo Köppen-Geiger de Sarricolea et al. (2017) & Modelo IGM \\
\hline Clima & & \\
\hline Temperatura & & \\
\hline Precipitaciones & & \\
\hline Vegetación & & \\
\hline Otros & & \\
\hline
\end{tabular}

Fuente: Elaboración propia.

Cierre: Los estudiantes deben presentar en grupo, mediante disertación (evaluación relacionada con las asignaturas de Lenguaje, Artes Visuales y Tecnología), los cambios observados más relevantes para la vida diaria en su entorno natural.

\section{Segunda actividad}

Inicio: Los estudiantes observan la figura 3 mostrada por el docente y luego deben trabajar en la sala de clases las siguientes actividades: 
1. Seleccionar una zona climática según macrozona geográfica de la figura 3.

2. Presentar un ejemplo explícito de un paisaje afectado por el cambio climático según los cambios señalados en la figura 4, en relación a su experiencia personal.

Desarrollo y cierre: Tras resolver las dos actividades anteriores, él o la docentes deben trabajar un efecto urbano del cambio climático para relacionar los contenidos. Por ejemplo, explicar y comunicar una visión e imagen a futuro de los paisajes urbanos afectados por el cambio climático en el territorio nacional, por medio de la imagen sobre la isla de calor urbana, según se aprecia en la figura 4. Esta metodología puede ser realizada desde una propuesta interdisciplinaria con la asignatura de Artes Visuales, en el diseño de una imagen a futuro; también se puede incluir aspectos de la informática con la utilización de software de rediseño, entre otras estrategias.

\section{Figura 3}

Condiciones de vulnerabilidad presentes en Chile, según establece la Convención Marco de las Naciones Unidas sobre el Cambio Climático

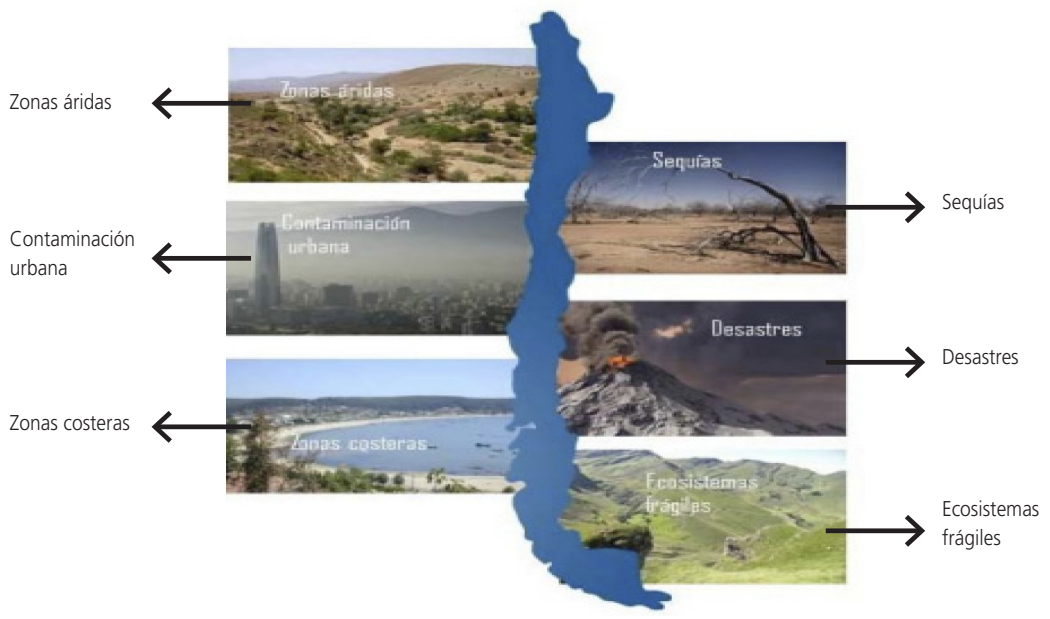

Fuente: http://portal.mma.gob.cl/wp-content/uploads/2018/06/Plan-CC-para-Ciudades_ aprobado-CMS-ene2018.pdf, revisado el 30-VI-2018. 


\section{Figura 4}

Isla de calor urbana

Cómo se produce el fenómeno

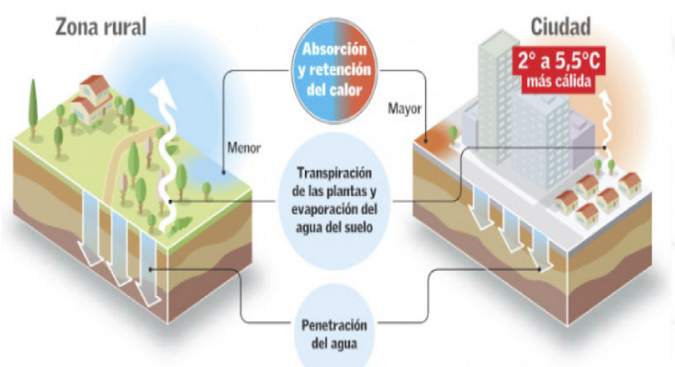

Santiago en una noche de verano

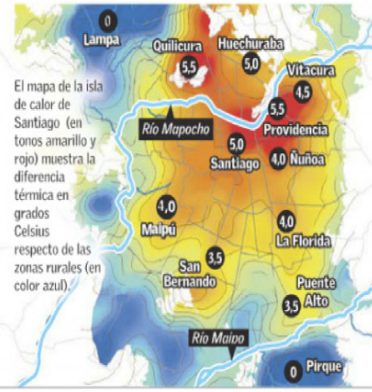

Fuente: http://www.plataformaurbana.cl/archive/2016/08/22/las-ciudades-se-ponen-cadavez-mas-calurosas-respecto-de-su-entorno-rural/, revisado el 30-VI-2018.

Recomendaciones: Estas dos actividades deben ser desarrolladas siguiendo la metodología de Köppen-Geiger, usado un trabajo de articulación docente (Ander-Egg, 1997) para lograr flexibilidad curricular en el tratamiento del contenido referente a cambio climático.

\section{Actividad 3}

\section{Actividad Propuesta}

Descripción de Plan de Formación Ciudadana para Enseñanza Media en el Centro Educacional Mariano Egaña de Peñalolén (CEME).

\begin{tabular}{|c|c|}
\hline $\begin{array}{l}\text { Departamento o } \\
\text { Asignatura }\end{array}$ & Historia, Geografía y Ciencias Sociales. \\
\hline $\begin{array}{l}\text { Acción (nombre o } \\
\text { descripción) }\end{array}$ & $\begin{array}{l}\text { Cambio climático en el aula actual, transformaciones del territorio } \\
\text { nacional y sus efectos en la vida de los estudiantes. } \\
\text { Plan de Mejoramiento Educativo (PME): Implementación de la } \\
\text { identidad Mariano Egaña en todos los niveles educativos, cuidado } \\
\text { del medio ambiente, difusión de los riesgos del cambio climático } \\
\text { para la comunidad educativa. }\end{array}$ \\
\hline Niveles & $1^{\circ}$ y $2^{\circ}$ medio. \\
\hline Objetivo & $\begin{array}{l}\text { Identificar las transformaciones y efectos que puede tener el cambio } \\
\text { climático en el país. }\end{array}$ \\
\hline \multirow{2}{*}{ Fechas } & Inicio: Agosto. \\
\hline & Término: Noviembre. \\
\hline
\end{tabular}




\begin{tabular}{|l|l|}
\hline Responsable (s) & Jefe de Departamento Ciencias Sociales. \\
\hline $\begin{array}{l}\text { Recursos para la } \\
\text { implementación }\end{array}$ & $\begin{array}{l}\text { Sala de clases, data, material cartográfico (mapas climáticos } \\
\text { de Chile), datos geográficos (sequía, precipitaciones, riesgos } \\
\text { socionaturales, eventos de catástrofe, recurrencia de fenómenos), } \\
\text { nuevo mapa climático de Chile Continental siguiendo el modelo } \\
\text { de Köppen-Geiger. }\end{array}$ \\
\hline $\begin{array}{l}\text { Financiamiento (si } \\
\text { requiere) }\end{array}$ & $\begin{array}{l}\text { Compra de nuevos mapas (\$100.000). } \\
\text { Medios de } \\
\text { verificación }\end{array}$ \\
\hline
\end{tabular}

Antes de generar la propuesta, es importante recordar que en las nuevas Bases Curriculares de la enseñanza media concernientes a Historia, Geografía y Ciencias Sociales las unidades referentes a Geografía prácticamente han desaparecido del currículum.

El docente debe mantener el objetivo propuesto en el Plan de Formación Ciudadana, el cual es promover la mejora permanente de todos los aprendizajes que involucra la formación ciudadana mediante espacios académicos, formativos, culturales y extracurriculares, con el fin de desarrollar en los estudiantes y en toda la comunidad escolar las habilidades y competencias necesarias para participar de manera constructiva y democrática en la sociedad actual. Fomentar en los estudiantes la tolerancia y respeto a la diversidad, equidad de género, prevención, vida sana, cuidado del medio ambiente, participación ciudadana y pluralismo (Plan de formación Ciudadana CEME, 2018). Para realizar esta implementación, es necesario que el docente actualice sus conocimientos desde la mirada de su quehacer académico geográfico, para que de esta manera sea vital transmitir los aprendizajes y obtener altos niveles de logros en los estudiantes. Esta situación nos hace repetir el concepto de articulación según Ander-Egg (1997), pero aplicando la nueva técnica de trabajo sobre los climas de Chile continental siguiendo la metodología de Köppen-Geiger en un contexto de cambio climático en el aula. Los colegios bajo el plan de mejoramiento educativo (PME) pueden establecer acciones (con los recursos que entrega el Estado) para actualizar sus fuentes de análisis, compras de materiales y materiales fungibles y no fungibles, que determinan un nuevo aprendizaje y actualización del curriculum que los docentes pueden no poseer.

\section{Conclusiones}

Dada la disminución de horas que ha experimentado la enseñanza de la Geografía en el currículum nacional, los docentes de esta disciplina están en desventaja en términos de horas-curso para poder enseñar estos 
contenidos. Según todos los cambios propuestos desde la autoridad estatal (Mensaje Presidencial, 2017), el tema del cambio climático es una prioridad nacional. Ante ello, se hace indispensable rediseñar las metodologías de aulas y acercar a los estudiantes a esta problemática, reforzando en ellos habilidades como el pensamiento crítico, análisis de fuentes y la discusión sobre el problema en sí.

En términos de actualizar las actividades, es posible revisar algunas propuestas para los niveles de enseñanza básica y reforzar el aprendizaje a través del acercamiento de los trabajos y las investigaciones que realizan las escuelas universitarias sobre el cambio climático. Por ejemplo, el uso de la nueva aplicación de los climas de Chile continental siguiendo la metodología de Köppen-Geiger en un contexto de cambio climático, se transforma en una herramienta metodológica para conocer, comprender y observar en el aula los cambios climáticos que afectan al territorio nacional en los últimos años. Desde esta perspectiva, este trabajo puede ser un avance para conocer una herramienta metodológica nueva para el uso y conocimiento de los cambios climáticos en el territorio nacional. Además, consideramos muy afín y necesario incluir en todo trabajo docente el concepto de articulación según lo expresado en las actividades propuestas.

En el caso del plan de formación ciudadana, recomendamos que en las escuelas que cuentan con la información para trabajar este tema, éste sea un avance en el desarrollo del trabajo educativo sobre la comprensión de la importancia de conocer las nuevas metodologías cartográficas en el área de la geografía actual, así como un incentivo de estudio que nace desde las escuelas para reforzar la relevancia de la enseñanza de la geografía en la sociedad y aulas actuales, destacando lo significativo de esta misma en la vida diaria de cada uno de nuestros estudiantes.

Por último, en concordancia con el profesor Capel (1998), las tres aristas a sus respuestas del por qué se debe enseñar la geografía, quedan refundadas en la importancia de un análisis climático actual del territorio nacional.

\section{Referencias bibliográficas}

\section{Fuentes primarias}

\section{a) Publicaciones oficiales}

Gobierno de Chile (2017). Mensaje Presidencial. s/e.

Ministerio de Educación (2013a). Programa de Estudio para Quinto Año Básico. Historia, Geografía y Ciencias Sociales. Santiago. 
Ministerio de Educación (2013b). Programa de Estudio para Sexto Año Básico. Historia, Geografía y Ciencias Sociales. Santiago.

Ministerio del Medio Ambiente (2012). Guía de apoyo docente en cambio climático. Santiago: Andros Impresores.

Centro Educacional Mariano Egaña (2018). Plan de Formación Ciudadana. Santiago: Unidad Técnica Pedagógica Básica y Media.

\section{Fuentes secundarias}

\section{a) Artículos y capítulos de libros}

Capel, H. (1998). "Una geografía para el siglo XXI", en Scripta Nova, Revista Electrónica de Geografía y Ciencias Sociales, Universidad de Barcelona, № 19, 15 de abril de 1998, http://www.ub.edu/geocrit/-xcol/19.htm, revisado el 5-VI-2018.

De la Calle, M. (2012). "La enseñanza de la geografía ante los nuevos desafíos ambientales, sociales y territoriales", en R. de Miguel, M. L. de Lázaro y Torres y M. J. Marrón (coords.). La educación geográfica digital. Zaragoza: Asociación de Geógrafos Españoles, Grupo de Didáctica de la Geografía (AGE), Universidad de Zaragoza, pp. 123-138.

De Miguel, R. (2014). "Aprendizaje por descubrimiento, enseñanza activa y geoinformación: hacia una didáctica de la geografía innovadora", en Didáctica Geográfica, № 14, pp. 17-36.

Garreaud, R. (2011). "Cambio Climático: Bases Físicas e Impactos en Chile", en Revista Tierra Adentro, № 93, pp. 13-19.

Gazmuri, R. (2017). "Ideologías curriculares en el debate y negociación del currículum chileno de Historia, Geografía y Ciencias Sociales", en Estudios pedagógicos, Vol. 43, № 1, pp. 157-169.

González, E., y Pablo Meira. (2009). "Educación, comunicación y cambio climático. Resistencias para la acción social responsable", en Trayectorias, Vol. 11, № 29, pp. 6-38.

Köppen, W. (1900a). "Versuch einer Klassifikation der Klimate, vorzugsweise nach ihren Beziehungen zur Pflanzenwelt" en Geographische Zeitschrift, Vol. 6, № 11, pp. 593-611.

Köppen, W. (1900b). "Versuch einer Klassifikation der Klimate, vorzugsweise nach ihren Beziehungen zur Pflanzenwelt (Schluss)" en Geographische Zeitschrift, Vol. 6, No 12, pp. 657-679.

Sarricolea P., María José Herrera y Oliver Meseguer (2017). "Climatic regionalisation of continental Chile", en Journal of Maps, Vol. 13, № 2, pp. 66-73. 
Souto, X. (2010). "¿Qué escuelas de Geografía para educar en ciudadanía?", en Didáctica de las ciencias experimentales y sociales, № 24, pp. 25-44.

Souto, X. (2018). "Una educación geográfica para el siglo XXI: Aprender competencias para ser ciudadano en el mundo global", en Anekumene, Vol. 1, $\mathrm{N}^{\circ}$ 1, pp. 28-47.

b) Libros

Ander-Egg, E. (1997). Diccionario Pedagógico. Argentina: Editorial Magisterio del Rio de la Plata.

\section{c) Otros}

Plan de adaptación al cambio climático para ciudades, 2018-2022. Aprobado por el Consejo de Ministros para la Sustentabilidad el 22 de Enero de 2018, http:// portal.mma.gob.cl/wp-content/uploads/2018/06/Plan-CC-para-Ciudades_aprobado-CMS-ene2018.pdf, revisado el 5-V-2018.

Sitio Plataforma Urbana, http://www.plataformaurbana.cl/archive/2016/08/22/ las-ciudades-se-ponen-cada-vez-mas-calurosas-respecto-de-su-entorno-rural/, revisado el 3-V-2018.

Sociedad Chilena de Ciencias Geográficas (2013). Informe de revisión de Bases Curriculares $7^{\circ}$ básico a $2^{\circ}$ medio. Santiago: s.le.

\section{Cómo citar este artículo:}

Sepúlveda Moreno, O. (2018) "Una propuesta pedagógica para el aprendizaje de la transformación climática de Chile en el contexto del cambio global", en Revista de Historia y Geografía, №39, pp. 121-141, Escuela de Educación en Historia y Geografía, Universidad Católica Silva Henríquez (UCSH), Santiago de Chile.

[http://ediciones.ucsh.cl/ojs/index.php/RHyG/article/view/1695/1557] 